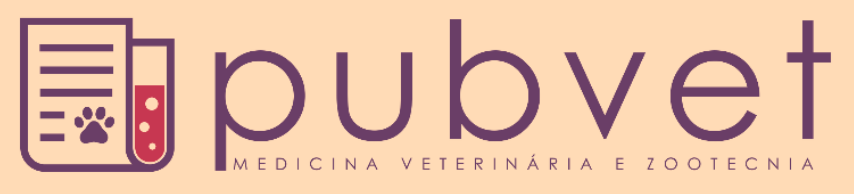

https://doi.org/10.31533/pubvet.v14n8a629.1-6

\title{
Protocolo anestésico para Sapajus libidinosus (macaco prego) submetidos a vasectomia e laqueadura de trompas eletivas
}

\author{
Thiago Vargas da Silva ${ }^{1 *}$, Marllos Henrique Vieira Nunes ${ }^{2} \bullet$, Juliana Tessália Wagatsuma ${ }^{2}$, \\ Thiago Martins $\operatorname{Souza}^{30}$, Alex Cardoso de Melo $^{3}{ }^{\circ}$, Amanda da Costa Andrade ${ }^{\circ}{ }^{\circ}$, Marcelo \\ Campos Rodrigues $^{30}$
}

${ }^{I}$ Residente hospital veterinário em Universidade Federal do Piauí. Teresina-PI Brasil.

${ }^{2}$ Professor em Universidade Federal do Piauí, área de Medicina Veterinária. Teresina-PI Brasil.

${ }^{3}$ Médico(a) veterinária, Teresina- PI Brasil.

*Autor para correspondência: E-mail: thiago_vargas@ @otmail.com

\begin{abstract}
Resumo. A captura, imobilização ou anestesia segura e efetiva de animais selvagens, como os macacos pregos (Sapajus libidinosus), geralmente é necessária para pesquisa e manejo, como também procedimentos cirúrgicos e diagnósticos, e dentre as espécies neotropicais, é a que apresenta maior distribuição geográfica. $O$ gênero vem sendo utilizado em diversas pesquisas, dada a sua proximidade filogenética com a espécie humana, servindo de modelo biológico em experimentos aplicados a essa espécie. Há uma variedade de protocolos anestésicos utilizados para que a captura e o manuseio de macacos pregos seja realizada de modo simples e seguro. $\mathrm{O}$ objetivo deste trabalho é relatar a utilização da associação de dexmedetomidina, cetamina, morfina e midazolam para a contenção química de oito macacos-prego adultos, submetidos a dois procedimentos cirúrgicos, vasectomia nos machos e laqueadura das trompas eletivas nas femêas, tendo como meta estabelecer um protocolo de imobilização e contenção química segura e eficaz para a espécie. Durante os procedimentos cirúrgicos foram monitorados os parâmetros fisiológicos de frequência cardíaca, frequência respiratória, oximetria de pulso e temperatura retal de oito macacos prego. Ao término das cirurgias, os animais receberam atipamezole por via intramuscular. O protocolo utilizado demonstrou-se seguro e eficaz para contenção química de macacosprego submetidos a procedimentos cirúrgicos distintos permitindo reversão dos efeitos farmacológicos ao término da cirurgia.
\end{abstract}

Palavras chave: Anestesia, animais selvagens, contenção, dissociativos, $\alpha$ 2-agonista

\section{Anesthetic protocol for Sapajus libidinosus (nail monkey) submitted to vasectomy and ligation of elective tubes}

\begin{abstract}
Safe and effective capture, immobilization or anesthesia of wild animals, such nail monkey (Sapajus lididinosus), is generally required for research and management purposes, as well as surgical and diagnostic procedures, and of the neotropical species, it is the one that presents greater geographical distribution. The genus has been used in several researches, given its phylogenetic proximity to the human species, serving as a biological model in experiments applied to this species There are a variety of anesthetic protocols used so that the capture and handling of nail monkey is performed simply and safely. The objective of this study is to report the use of the combination of dexmedetomidine with ketamine, morphine and midazolam for the chemical restraint of eight adult nail monkeys submitted to two surgical procedures, to vasectomy and ligation of elective tubes, with the goal of establishing a safe and effective chemical containment and containment protocol for the species in captivity. During the surgical procedures the
\end{abstract}


physiological parameters of heart rate and respiratory rate and rectal temperature and oxyhemoglobin saturation by pulse oximetry of the eight nail monkeys were monitored. At the end of the surgeries, the animals received atipamezole intramuscularly. The protocol used was safe and effective for the chemical containment of nail monkeys in captivity submitted to different surgical procedures allowing reversal of the pharmacological effects at the end of the surgery.

Keywords: $\alpha 2$-agonist, anesthesia, wild animals dissociative, immobilization

\title{
Protocolo anestésico para Sapajus libidinosus (monos capuchinos robustos) sometido a vasectomía y ligadura de trompas
}

\begin{abstract}
Resumen. La captura, inmovilización o anestesia segura y efectiva de animales salvajes, como monos capuchinos robustos (Sapajus libidinosus), suele ser necesaria para la investigación y el manejo, así como los procedimientos quirúrgicos y de diagnóstico, y entre las especies neotropicales, es la que tiene una mayor distribución geográfica. El género se ha utilizado en varios estudios, dada su proximidad filogenética a la especie humana, sirviendo como modelo biológico en experimentos aplicados a esta especie. Hay una variedad de protocolos anestésicos utilizados para capturar y manipular monos capuchinos robustos de una manera simple y segura. El objetivo de este trabajo es informar el uso de la asociación de dexmedetomidina, ketamina, morfina y midazolam para la contención química de ocho monos adultos, sometidos a dos procedimientos quirúrgicos, vasectomía en machos y ligadura de trompas en las hembras, con la finalidad de establecer un protocolo de inmovilización y contención química segura y eficaz para la especie. Durante los procedimientos quirúrgicos, se monitorizaron los parámetros fisiológicos de la frecuencia cardíaca, la frecuencia respiratoria, la oximetría de pulso y la temperatura rectal de los ocho monos. Al final de las cirugías, los animales recibieron atipamezol intramuscular. El protocolo utilizado demostró ser seguro y eficaz para la contención química de monos capuchinos robustos sometidos a diferentes procedimientos quirúrgicos que permiten la reversión de los efectos farmacológicos al final de la cirugía.
\end{abstract}

Palabras clave: Anestesia, animales salvajes, contención, disociativa, agonista $\alpha 2$

\section{Introdução}

O Sapajus libidinosus, conhecido popularmente como macaco prego, é uma das espécies mais abundantes do Brasil. Dentre as espécies neotropicais, é a que apresenta maior distribuição geográfica. Além disso, é um primata não humano com alta adaptabilidade e de fácil reprodução em cativeiros e áreas que tenham sofrido alterações pelos processos humanos (Ludwig et al., 2005), resultando em grandes populações. Com isso, seu controle populacional se dá por meio de intervenção cirúrgica, através de vasectomia e laqueadura das trompas, que se tornam técnicas eficazes, e que acabam por preservar o comportamento natural da espécie, não alterando a hierarquia no grupo. A alteração e a destruição sistemáticas dos habitats naturais promovidas pelos seres humanos têm levado ao aumento da competição entre humanos e animais silvestres por espaço e recursos (Distefano, 2005).

A contenção química destes animais se faz necessária pela dificuldade de realizar-se uma avaliação pré-anestésica, diante do comportamento de fuga, força e agitação (Aquino, 2005; Grimm et al., 2015), como também a obtenção de planos anestésicos que possibilitem a realização do procedimento.

Existe uma variedade de protocolos anestésicos para que a captura e o manuseio do Sapajus libidinosus (macaco prego) possa ser realizada de modo simples e seguro, haja vista que o índice de mortalidade de pequenos mamíferos é maior durante a sedação ou anestesia do que em animais domésticos (cães, gatos, coelhos, etc), sendo os cuidados para anestesiar essas espécies mais intensos (Brodbelt et al., 2007). Com isso a contenção química torna-se indispensável e o emprego de protocolos anestésicos efetivos e seguros a integridade animal são de extrema importância. 
Diante do exposto, objetivou-se com esse trabalho, estabelecer um protocolo de anestesia balanceada que propicie contenção química, analgesia trans e pós-operatória, baixo requerimento anestésico e rápida recuperação dos pacientes da espécie supracitada.

\section{Relato de caso}

Oito macacos pregos (Sapajus libidinosus), sendo quatro machos e quatro fêmeas, sexualmente maduros, pesando 3,17 $\pm 1,39 \mathrm{~kg}$, pertencentes ao Parque Zoobotânico de Teresina, Piaú, foram encaminhados ao Hospital Veterinário Universitário da Universidade Federal do Piauí para a realização de procedimentos cirúrgicos de vasectomia e laqueadura de trompas. Estes animais foram contidos fisicamente com o auxílio de um puçá e como medicação pré-anestésica, foi administrado $7 \mu \mathrm{g} / \mathrm{kg}$ de dexmedetomidina (dextomidor ${ }^{\circledR}, 0,5 \mathrm{mg} / \mathrm{mL}$ ) associada a $7 \mathrm{mg} / \mathrm{kg}$ de cetamina (Quetamina ${ }^{\circledR}, 100$ $\mathrm{mg} / \mathrm{mL}$ ), em três animais, e $3 \mu \mathrm{g} / \mathrm{kg}$ de dexmedetomidina (dextomidor ${ }^{\circledR}, 0,5 \mathrm{mg} / \mathrm{ml}$ ) associada à 3 $\mathrm{mg} / \mathrm{kg}$ de cetamina (Quetamina ${ }^{\circledR}, 100 \mathrm{mg} / \mathrm{ml}$ ) em cinco macacos, sendo essas doses associadas com 0,5 $\mathrm{mg} / \mathrm{kg}$ de midazolam e $0,1 \mathrm{mg} / \mathrm{kg}$ de morfina, administrados por via intramuscular, no músculo semimembranoso dos membros pélvicos dos animais. Não fora realizado o exame físico geral prévio e laboratorial (hemograma e bioquímico) por motivos de segurança e comportamento imprevisível e agressivo dos macacos prego.

Após 5 minutos, os animais apresentaram sedação e relaxamento intensos, em seguida realizou-se a cateterização da veia cefálica com cateter $24 \mathrm{G}$ para fluidoterapia de manutenção com solução de ringer com lactato $(5 \mathrm{ml} / \mathrm{kg} / \mathrm{hora})$.

Os macacos foram intubados com sonda endotraqueal $n^{\circ} 3.5$, somente após a dessensibilização laríngea, com instilação tópica de $1 \mathrm{mg} / \mathrm{kg}$ de lidocaína sem vasoconstritor a $2 \%$, iniciando assim o fornecimento de oxigênio a $100 \%$ e de isoflurano numa concentração anestésica de $0,75 \% \pm 0,13 \%$, via circuito tipo Baraka em ventilação espontânea ,além do início da monitoração anestésica (frequência cardíaca, saturação de oxihemoglobina e temperatura retal) com base na figura 1.

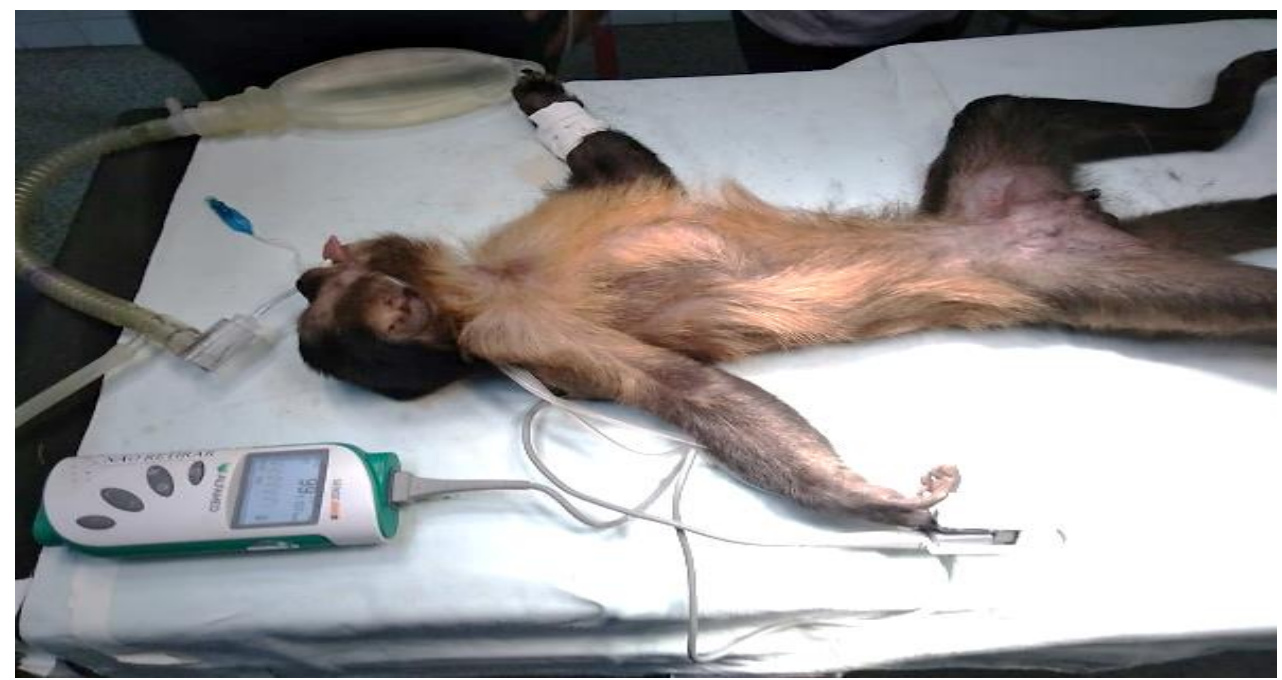

Figura 1. Sensor de oximetria de pulso (SENSE 10, ALFAMED ${ }^{\circledR)}$ posicionado em falanges do membro torácico esquerdo em (Sapajus libidinosus), macho, adulto, Teresina.

No caso dos machos de macaco prego foi realizado o bloqueio do cordão espermático, utilizando-se a dose de $0,25 \mathrm{ml} / \mathrm{kg}$ de lidocaína sem vasoconstritor, o que corresponde a dose de $5 \mathrm{mg} / \mathrm{kg}$ por animal.

Os tempos cirúrgicos para vasectomia e laqueadura de trompas foi de $20 \pm 4,08$ e 23,75 \pm 7,5 minutos, respectivamente. A média dos parâmetros vitais (frequência cardíaca, respiratória, saturação de oxihemoglobina e temperatura retal) dos animais submetidos a doses maiores de dexmedetomidina e cetamina quando comparado aos que receberam doses menores foram semelhantes.

Finalizadas as cirurgias, aplicou-se o antagonista de fármacos agonistas de receptores $\alpha$-2 adrenérgicos, o atipamezole (Antisedan ${ }^{\circledR} 0,5 \%$, Zoetis), na dose de $30 \mu \mathrm{g}-1 \mathrm{~kg}-1$, por via intramuscular, na região do músculo semimembranoso. 
Em seguida, os animais foram transferidos para as gaiolas de transporte e posicionados em decúbito esternal, e acompanhados por anestesistas até à movimentação e a recuperação anestésica. $\mathrm{O}$ tempo de recuperação referiu-se ao intervalo entre o momento da administração do antagonista até momento que os macaco pregos assumiram posição quadrupedal e respostas de consciência satisfatória.

O tempo de recuperação anestésica nas doses maiores de dexmedetomidina e cetamina foi significativamente superior quando comparado aos que receberam doses menores dos mesmos fármacos $(86,75 \pm 27,68$ e $12,75 \pm 4,11$ minutos, respectivamente) .

\section{Resultados}

De acordo com o protocolo utilizado, foram necessários em média cinco minutos para que os macacos assumissem decúbito esternal e imobilidade, permitindo aproximação e manejo para preparo cirúrgico, apesar que a captura dos animais para administração das drogas foi lenta e estressante, mas comprovaram estudos utilizando $10 \mu \mathrm{g}-1 \mathrm{~kg}-1$ de dexmedetomidina (dextomidor ${ }^{\circledR}, 0,5 \mathrm{mg} / \mathrm{mL}$ ) e 10 mg-1 kg- de cetamina (Quetamina ${ }^{\circledR}, 100 \mathrm{mg} / \mathrm{ml}$ ), em macaco pregos que tiveram o mesmo tempo para alcançar imobilidade e decúbito (Disarz et al., 2019). Ainda que os animais sejam submetidos a grandes manipulações, causando estresse e mudança de comportamento podendo afetar ou até mesmo anular o tempo de latência do anestésico para manipulação, principalmente pela liberação de catecolaminas (Theriault et al., 2008).

Não foi observado êmese, bradicardia, arritmia ou bradpneia, que são efeitos já observados em estudos utilizando as doses de $30 \mu \mathrm{g} / \mathrm{kg}$ e $50 \mu \mathrm{g} / \mathrm{kg}$ de dexmedetomidina (dextomidor ${ }^{\circledR}, 0,5 \mathrm{mg} / \mathrm{mL}$ )) associada a 7,5 mg de cetamina (Quetamina ${ }^{\circledR}, 100 \mathrm{mg} / \mathrm{ml}$ ) (Chagas et al., 2018).

Apesar do pequeno porte, a canulação da veia cefálica em macacos prego é um procedimento simples, sendo mais importante a correta fixação do cateter e manutenção do fluxo da fluidoterapia.

A qualidade da indução foi excelente, se deu de forma tranquila e sem mais intercorrências, os animais foram induzidos em mascara anestésica, utilizando-se isoflurano a $5 \%$ em vaporizador calibrado como fornecimento de oxigênio a $100 \%$, não foi observado hipersalivação, sendo um sinal já observado na associação de dexmedetomidina e cetamina (Selmi et al., 2004).

Após a indução em mascara, onde observava-se a ausência de respostas motoras, rotação de globo ocular, relaxamento de mandíbula e membros, prosseguíamos com o processo de intubação, mesmo que os primatas não-humanos não apresentem dificuldade de visualização da laringe, há possibilidade de colocarmos o animal em posição esternal, mesmo apresentando uma maior angulação temporomandibular e as sondas endotraqueais apresentando uma alta flexibilidade, tornando o processo mais difícil. O mesmo foi realizado após instilarmos $1 \mathrm{mg} . \mathrm{kg}$ de lidocaína sem vasoconstritor a $2 \%$ para dessensibilização da laringe (Flecknell, 2008), utilizando-se uma sonda endotraqueal número 3,5. Não houve intercorrências durante o processo.

A utilização de anestésicos inalatórios se torna imprescindível em processos mais longos, sendo muito recomendado em primatas não-humanos, tanto para indução quanto para manutenção anestésica, a concentração recomendada para indução em mascara anestésica é de $1 \%$ a $2 \%$ de isoflurano, para manutenção anestésica recomenda-se de $0,7 \%$ a 1,5\% (Vasconcelos et al., 2000). A concentração anestésica dos relatos, foram de $1 \%$, podendo chegar a $0,7 \%$ de isoflurano vaporizado. Em seguida começamos a monitoração anestésica dos macacos pregos.

O bloqueio de cordão espermático e uma técnica antiga, sendo bastante utilizada para primatas nãohumanos e descrita como de fácil execução (Fuchs, 1982). No presente relato, foi executado a técnica a cega, que consistia na palpação manual e identificação do cordão espermático, prosseguindo com a inserção da agulha na região identificada para injetarmos o anestésico local, esse tipo de técnica possui algumas vantagens, tais como: redução do consumo de opioides e maior controle analgésico durante o trans cirúrgico, mas também pode possuir algumas desvantagens, principalmente o que diz a injeção de anestésico de forma intravascular, lesões vasculares e danos aos ductos deferentes. Essas desvantagens podem se tornar nulas, mediante uso de aparelho de ultrassonografia, para correta identificação do cordão espermático (Gordon \& Rifenburg, 2016). No presente relato não houve nenhuma intercorrência. 
A média dos parâmetros vitais (frequências cardíaca e respiratória, saturação de oxihemoglobina e temperatura retal) dos animais submetidos a doses maiores de dexmedetomidina e cetamina quando comparado aos que receberam doses menores foram semelhantes. A temperatura corporal tratava-se de uma grande preocupação para a equipe, pois ocorre relaxamento muscular, termolise, ação dos fármacos anestésicos, ambiente, tempo cirúrgico e condições clinicas do animal que poderiam afetar o controle térmico. Ainda pode somar-se o efeito vasodilatador causado pelo isoflurano, que acaba por se tornar um determinante no processo de hipotermia, mas como foi utilizado um colchão térmico, que possui um controle térmico bem rigoroso, não tivemos intercorrências.

Mesmo com a utilização de alfa 2 agonistas, não foi observado bradicardia, que acontece simultaneamente com o aumento da pressão arterial (Paris \& Tonner, 2005; Selmi et al., 2004), em todos os animais a frequência cardíaca se manteve estável, com uma média entre os dois grupos sem diferença significativa estatisticamente. Decréscimo de frequência respiratória e comum quando se faz o uso de alfa 2 agonistas, principalmente quando associados a outros fármacos, como propofol, cetamina ou opioides, eles podem causar severa redução do volume minuto, o que poderia causar injurias por hipóxia muito graves. Não foi observada hipoxemia. A saturação da hemoglobina deve estar entre 95 e $99 \%$, e valores abaixo de $94 \%$ denotam hipoxemia nos macacos pregos (Grimm et al., 2015; $\underline{\text { Votava et al., }}$ 2011).

O tempo de recuperação anestésica nas doses maiores de dexmedetomidina e cetamina foi significativamente superior quando comparado aos que receberam doses menores dos mesmos fármacos $(86,75 \pm 27,68$ e 12,75 $\pm 4,11$ minutos, respectivamente). Os animais submetidos à anestesia dissociativa podem exibir comportamento exacerbado durante a recuperação, incluindo percepção errônea de estímulos auditivos e visuais, ataxia, atividade motora aumentada e hiper-reflexia (Galante et al., 2014).

A boa recuperação da medicação pré-anestésica para cetamina e dexmedetomidina foi semelhante aos achados de Chagas et al. (2018) que usaram o mesmo protocolo em macacos bugios.

\section{Conclusão}

O protocolo anestésico para procedimentos de vasectomia e laqueadura de trompas foi eficiente para a contenção química dos Sapajus libidinosus, proporcionando sedação e relaxamento muscular, intubação orotraqueal, analgesia e estabilidade dos parâmetros fisiológicos analisados, sendo seguro na espécie, entretanto os animais submetidos às doses menores de dexmedetomidina e cetamina apresentaram recuperação anestésica de melhor qualidade, enquanto que a contenção química, o plano anestésico e os parâmetros vitais foram equivalentes aos animais que receberam doses maiores destes mesmos fármacos.

Conclui-se que os protocolos apresentados apresentam segurança para a realização na espécie, atentando-se ao tempo de procedimento a ser realizado. Com base nisso podemos definir um protocolo de tempo reduzido para procedimentos mais simples, e o de lenta recuperação para procedimentos mais invasivos.

\section{Referências}

Aquino, N. M. (2005). Manual de vigilância de epizootias em primatas não humanos. In Secretaria de Vigilância em Saúde. Ministério da Saúde.

Brodbelt, D. C., Pfeiffer, D. U., Young, L. E., \& Wood, J. L. N. (2007). Risk factors for anaestheticrelated death in cats: results from the confidential enquiry into perioperative small animal fatalities (CEPSAF). British Journal of Anaesthesia, 99(5), 617-623. https://doi.org/10.1093/bja/aem229

Chagas, J. A. B., Santos, L. C. P., Silva Filho, J. R., \& Bondan, C. (2018). Anaesthetic and cardiorespiratory effects of ketamine plus dexmedetomidine for chemical restraint in black capuchin monkeys (Sapajus nigritus). New Zealand Veterinary Journal, 66(2), 79-84. https://doi.org/10.1080/00480169.2017.1403975

Disarz, P., Adams, P. P., Carvalho, A. L., Wu, S., Lima, C. L., \& Fukushima, F. B. (2019). Anestesia geral em Sapajus nigritus (Macaco-prego). Acta Scientiae Veterinariae, 47(Sup. 1), 368.

Distefano, E. (2005). Human-Wildlife Conflict worldwide: collection of case studies, analysis of 
management strategies and good practices. In Food and Agricultural Organization of the United Nations.

Flecknell, P. A. (2008). Analgesia from a veterinary perspective. British Journal of Anaesthesia, 101(1), 121-124. https://doi.org/10.1093/bja/aen087

Fuchs, E. F. (1982). Cord block anesthesia for scrotal surgery. The Journal of Urology, 128(4), 718719. https://doi.org/10.1016/S0022-5347(17)53153-5

Galante, J., Galante, I., Bekkers, M.-J., \& Gallacher, J. (2014). Effect of kindness-based meditation on health and well-being: a systematic review and meta-analysis. Journal of Consulting and Clinical Psychology, 82(6), 1101-1114. https://doi.org/10.1037/a0037249

Gordon, J., \& Rifenburg, R. P. (2016). Spermatic cord anesthesia block: an old technique re-imaged. Western Journal of Emergency Medicine, 17(6), 811-813. https://doi.org/10.5811/westjem.2016.8.31017

Grimm, K., Lamont, L., Tranquilli, W., Greene, S., \& Robertson, S. (2015). Anestesiologia e analgesia em veterinária. Editora Roca.

Ludwig, G., Aguiar, L. M., \& Rocha, V. J. (2005). Uma avaliação da dieta, da área de vida e das estimativas populacionais de Cebus nigritus (Goldfuss, 1809) em um fragmento florestal no norte do estado do Paraná. Neotropical Primates, 13(3), 12-18. https://doi.org/10.1896/1413-4705.13.3.12

Paris, A., \& Tonner, P. H. (2005). Dexmedetomidine in anaesthesia. Current Opinion in Anesthesiology, 18(4), 412-418. https://doi.org/10.1097/01.aco.0000174958.05383.d5

Selmi, A. L., Mendes, G. M., Boere, V., Cozer, L. A. S., Emivaldo Filho, S., \& Silva, C. A. (2004). Assessment of dexmedetomidine/ketamine anesthesia in golden-headed lion tamarins (Leontopithecus chrysomelas). Veterinary Anaesthesia and Analgesia, 31(2), 138-145. https://doi.org/10.1111/j.1467-2987.2004.00147.x

Theriault, B. R., Reed, D. A., \& Niekrasz, M. A. (2008). Reversible medetomidine/ketamine anesthesia in captive capuchin monkeys (Cebus apella). Journal of Medical Primatology, 37, 74-81. https://doi.org/10.1111/j.1600-0684.2007.00267.x

Vasconcelos, C. H. de C., Mársico Filho, F., Segura, I. A. G., Nascimento, P. R. L., \& Mpnteiro, R. V. (2000). Use of isoflurane in capuchin monkeys (Cebus apella-Cebidae, Primata). Brazilian Journal of Veterinary Research and Animal Science, 37(1), 65-69.

Votava, M., Hess, L., Schreiberová, J., Málek, J., \& Štein, K. (2011). Short term pharmacological immobilization in macaque monkeys. Veterinary Anaesthesia and Analgesia, 38(5), 490-493. https://doi.org/10.1111/j.1467-2995.2011.00637.x

Recebido: 5 de março, 2020.

Aprovado: 1 de abril, 2020.

Disponivel online: 17 de julho, 2020.

Licenciamento: Este artigo é publicado na modalidade Acesso Aberto sob a licença Creative Commons Atribuição 4.0 (CC-BY 4.0), a qual permite uso irrestrito, distribuição, reprodução em qualquer meio, desde que o autor e a fonte sejam devidamente creditados. 